\section{AB0931 TRANSLATION AND VALIDATION OF THE MTX INTOLERANCE SEVERITY SCORE QUESTIONNAIRE FOR PORTUGUESE VERSION IN BRAZIL IN JUVENILE IDIOPATHIC ARTHRITIS}

Ana Carolina Londe, Roberto Marini, Simone Appenzeller. University of Capinas, Campinas, Brazil

Background: Methotrexate (MTX) is the first-choice disease-modifying antirheumatic drug (DMARD) for the treatment of juvenile idiopathic arthritis (JIA). During therapy, there are frequent reports of discontinuation of MTX, either by physicians or patient's own conduct. Through the MTX Intolerance Severity Score questionnaire (MISS), we determined the prevalence of MTX intolerance.

Objectives: Translate and validate the MTX Intolerance Severity Score questionnaire (MISS) to Portuguese.

Methods: The MISS was translated into Portuguese following the "Guidelines for the process of cross-cultural adaptation of self-report measures". The MISS consists of 4 domains: stomachache, nausea, vomiting, and behavioral complaints. Each domain includes three to four items, and for every item, four answers are possible: no complaints (0), mild complaints (1 point), moderate complaints (2 points), and severe complaints ( 3 points). The points are summed to give a total score from 0 to 36 . Statically analysis was performed on the SPSS ${ }^{\oplus}$, version 21 . The psychometric properties were analyzed according to the Consensus based Standards for the Selection of Health Measurement Instruments (COSMIN), analyzing acceptability for each item; internal consistency using Cronbach's alpha coefficient and reproducibility assessed by Kappa. We plot the ROC curve to evaluate the discriminant validity of the MISS compared to gold standard (clinical interview) and cut-off score was determined.

Results: We included 220 subjects, 144 patients with JIA in use for least 3 months of MTX and 76 parents. The median age of patients were 18.3 SD \pm 8.7 years. Seventy-three $(73 \%)$ patients were females and the JIA subtype most frequently observed was polyarticular. Routes of administration of MTX were subcutaneous (81\%) and oral (19\%). All the subjects answered the MISS with less than 5 minutes. The internal consistency of MISS had a Cronbach's alpha $=0.851$ (patients) and 0.805 (parents), considered good $(\geq 0.8)$. The reproducibility between the test (40 patients) and the retest done after 15 days ( 36 patients) was almost perfect (kappa> 0.8). Reliability between patients and parents was almost perfect (kappa> 0.8), except stomachache (anticipatory with kappa $=0.30)$; considered weak $(\kappa=0.2-0.4)$ and stomachache by association $(\kappa=0.54)$; considered moderate $(\kappa=0.4-0.6)$. A cut-off scores of 3 showed the best sensitivity (93\%) and specificity $(71 \%)$. Using this cutoff we observed $78(54.2 \%)$ patients intolerant

Conclusion: MISS is a good tool for physicians, because it can not only measure the intolerance, but also explore the different forms in which it manifests. Although MTX has a great therapeutic index, the adverse reactions are still seen as a major form of abandonment of this pharmacological treatment. Therefore a careful history is essential to identify side effects and adequate treatment to increase adherence.

\section{REFERENCES}

1] Bulatovic M, et al. Arthritis\&amp:Rheumatism. 2011; 63: 2007-2013.

[2] Chausset A, et al. Clin Rheumatol. 2017; 36:1281-1288.

[3] Beaton D, et al. Spine.2000; 24:3186-3191.

[4] Terwee C, et al. J Clin Epidemiol.2007; 60:34-42.

Disclosure of Interests: None declared

DOI: 10.1136/annrheumdis-2019-eular.6077

\section{\begin{tabular}{l|l} 
AB0932 & PREDICTORS OF FLARE FOLLOWING ETANERCEPT
\end{tabular} WITHDRAWAL IN PATIENTS WITH RHEUMATOID FACTOR NEGATIVE JUVENILE IDIOPATHIC ARTHRITIS DURING 48 MONTHS OF FOLLOW-UP}

Angela Aquilani ${ }^{1}$, Denise Pires Marafon ${ }^{2}$, Andrea Uva ${ }^{1}$, Hanan Jadoun ${ }^{1}$, Fabio Basta ${ }^{1}$, Rebecca Nicolai ${ }^{1}$, Fabrizio De Benedetti ${ }^{1}$, Silvia Magni-Manzoni ${ }^{1}$. ${ }^{1}$ IRCCS Ospedale Pediatrico Bambino Gesù, Division of Rheumatology, Roma, Italy; ${ }^{2}$ Fondazione IRCCS Ca' Grande Ospedale Maggiore Policlinico, Intermediate Pediatric Care Unit, Milan, Italy

Background: Little is known about the risk of flare after etanercept (ETN) withdrawal in children with Juvenile Idiopathic Arthritis (JIA). Recently we conducted a retrospective chart review of 110 patients with JIA who discontinued ETN due to persistent clinically inactive disease (CID) on medication and were followed up to 12 months after ETN withdrawal. We showed that $60 \%$ of patients flared with arthritis. Male gender, presence of ANA and elevated CRP at baseline were associated with higher risk of flare.

Objectives: To evaluate frequency and timing of flares during 48 months of follow-up after withdrawal of ETN first course in patients with JIA who attained clinical remission on medication and to identify predictors of early flares ( $<12$ months after withdrawal of ETN first course) and predictors of repeated flares $(\geq 2)$.

Methods: The 110 patients enrolled in the previous study with oligo (o$\mathrm{JIA}$ ) and RF-negative polyarticular JIA ( $p$-JIA) who received a first course of ETN for at least 18 months and maintained clinically inactive disease (CID) for at least 6 months on treatment, were followed for 48 months after withdrawal of first course of ETN. We excluded patients that switched or swapped to other biologics or with limited follow-up. Demographic and clinical features at onset, at baseline (initiation of ETN) and at time of disease flares were collected.

Results: Among the cohort of the study, 10 patients (9\%) were lost to follow-up and $18(16 \%)$ switched/swapped to other biologics during 48 months of follow-up after withdrawal of ETN first course. Of the 82 patients included in the analysis, $55(67 \%)$ were treated with only one course of ETN, while $27(33 \%)$ received more than one course of ETN. The median age at disease onset was 3.6 years (IQR 1.9-8.5) and $72 \%$ of patients were female, ANA positive patients had a younger age at onset and were more frequently 0-JIA. After withdrawal of ETN first course, 70 of the $82(85 \%)$ patients enrolled flared with arthritis, without evident differences between o-JIA and p-JIA. Disease duration at diagnosis and at ETN start, total number of joints involved and total MTX treatment duration were not associated with flare. Patients who flared were more frequently males $(p=0.017)$ and ANA positive $(p=0.026)$, presented with higher levels of CRP $(p=0.029)$ at baseline and had longer total ETN treatment duration $(\mathrm{p}=0.0046)$. Higher values of CRP were associated with a higher risk of repeated flares $(p=0.0094)$. No predictors of early flares were identified in this group.

When we analysed the 55 patients treated with one course of ETN, 48 (87\%) flared during follow-up. Patients who flared were more frequently ANA positive $(p=0.015)$, presented higher CRP levels $(p=0.042)$ at baseline and concomitant MTX at ETN withdrawal $(p=0.009)$. Higher levels of CRP was also associated with higher numbers of repeated flares $(p=0.049)$. No predictors of early flares were identified in this subgroup. Conclusion: Our results show that almost $90 \%$ of patients with JIA experience at least one flare after ETN withdrawal during 48 months of follow-up. Our findings confirmed that male gender, positive ANA and elevated CRP at baseline are associated with a higher risk of flare. Higher CRP levels at baseline resulted also associated with a higher risk of repeated flares.

\section{REFERENCES}

[1] Predictors of Flare Following Etanercept Withdrawal in Patients with Rheumatoid Factor-negative Juvenile Idiopathic Arthritis Who Reached Remission while Taking Medication.

[2] Aquilani A, Marafon DP, Marasco E, Nicolai R, Messia V, Perfetti F, Magni-Manzoni S. J Rheumatol. 2018;45:956-961

Disclosure of Interests: Angela Aquilani: None declared, Denise Pires Marafon: None declared, Andrea Uva: None declared, Hanan Jadoun: None declared, Fabio Basta: None declared, Rebecca Nicolai: None declared, Fabrizio De Benedetti Grant/research support from: Abbvie, SOBI, Novimmune, Roche, Novartis, Sanofi, Pfizer, Silvia Magni-Manzoni Consultant for: Abbvie, Speakers bureau: Abbvie

DOI: 10.1136/annrheumdis-2019-eular.5721

\section{AB0933 ANALYSIS OF THE STATE OF IMMUNITY IN PATIENTS WITH JIA IN TREATMENT}

Inmaculada Concepcion Aranda-Valera ${ }^{1,2}$, Ladehesa Pineda Lourdes ${ }^{1}$, Font Ugalde Pilar ${ }^{2}$, Fernández de la Puebla Lechuga Elena ${ }^{3}$, Copete Marín Sara ${ }^{4}$ Eduardo Collantes Estevez ${ }^{1,2}$, Roldán Molina Rosa ${ }^{1} .{ }^{1}$ Reina Sofía University Hospital, Rheumatology Service, Córdoba, Spain; ${ }^{2}$ University Of Córdoba (UCO), Medicine, Córdoba, Spain; ${ }^{3}$ Reina Sofía University Hospital, Pediatric, Córdoba, Spain; ${ }^{4}$ Valle de los Pedroches Regional Hospital, Rheumatology Service, Córdoba, Spain

Background: The correct indication of the suspension of treatment with FAMES and biological therapy in pediatric patients, in the presence of a mild infectious disease or vaccination is a common problem in children's rheumatology. In patients with juvenile idiopathic arthritis (JIA), joint or extra-articular clinical reactivation during these "pauses" is not uncommon, 
however, there are not enough studies in the pediatric population to show the influence of treatments on the immune system., and therefore, that justify this clinical practice.

Objectives: To analyze the levels of T, B and NK lymphocyte subpopulations in patients diagnosed with JIA treated with FAMES and biological therapy.

Methods: A descriptive and cross-sectional study in which 39 patients from the Pediatric Rheumatology Unit of the Reina Sofía University Hospital were recruited, diagnosed with JIA according to the ILAR 2001 criteria. The patients were divided into four groups: 8 controls in clinical remission without treatment, 17 in treatment with DMARD in monotherapy, 7 in biological treatment in monotherapy and 7 in treatment with DMARD-biological. Patients with systemic JIA were excluded because they had a pathogenic mechanism different from the rest of the JIA categories. By flow cytometry, the levels of CD3, CD4, CD8 and CD19 cells were measured for acquired immunity and from NK for innate, the CD4/ CD8 index was also calculated.

Results: The mean age of the 39 patients was $10 \pm 5.7$ years, 29 were girls $(74.3 \%), 4$ patients had arthritis related to enthesitis, 16 patients had oligoarticular involvement ANA +, 6 subjects polyarticular involvement FR- and 13 they were psoriatic arthritis. Although no statistically significant differences were found when contracting cellular levels among the 4 groups evaluated, it was observed that the group treated with DMARD monotherapy had the highest percentage of children with alterations in cellular levels CD3, CD4, CD8 and CD19 (41.17\% of the patients of the group); the group treated in monotherapy with biological treatment $(28 \%)$ presented alteration in the levels of CD8 and CD19 and the group treated in combination of DMARD and biological (14.28\%) in CD19. On the other hand, the NK cells and the CD4/CD8 index were not altered in any of the groups. Only 6 cases of serious infections were registered in patients in combination therapy (DMARD-biologic) who had received corticosteroids by clinical activity. There were no statistical differences between patients who had received corticosteroids and those who did not.

Conclusion: Patients in treatment with DMARD monotherapy had a tendency to decrease cellular levels. On the other hand, alterations in innate immunity or CD4/CD8 index were not observed.

Disclosure of Interests: None declared

DOI: 10.1136/annrheumdis-2019-eular.3753

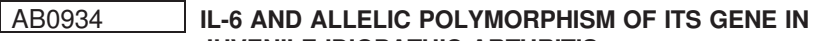 JUVENILE IDIOPATHIC ARTHRITIS}

Agar Artsymovych, Olena Oshlianska. Shupyk National Medical Academy of Postgraduate Education, Paediatrics №1, Kyiv, Ukraine

Background: The development and maintenance of inflammation in juvenile idiopathic arthritis (JIA) is mediated by cytokine imbalance; interleukin 6(IL6) plays a leading role among pro-inflammatory cytokines. Its pathological synthesis has a negative impact on all organs and system. It is not excluded that its effector ability depends on genetic structures of IL6 gene. It has not been studied whether the allelic polymorphism of the IL6-174CG gene affects the effectiveness of targeted biological therapy. Objectives: to assess the IL6 dynamics level in serum of patients with ineffective JIA-treatment.

Methods: The level of IL6 in the serum of JIA patients 1-18y.o. was determined using ECLIA method, debut of the disease (54) and treatment failure (36: 6 oJIA, 14 pJIA, 10 enthJIA, 6 sJIA). In patients with JIA, allelic polymorphism of the IL6-174CG gene was studied by PCR-method using allele-specific primers. A correlation analysis of clinical and laboratory parameters was made.

Results: Among patients with ineffective treatment of JIA, the duration of the disease was $39.5 \pm 35.8$ months, $62.9 \%$ were girls. 27 patients received GC (<1 mg/kg), 30-MTX(10-15mg/m2), 3-leflunomide, 1-AZA, 10TZ, 16-ADA/ETA, 1-TFC, 5 were switched from antiTNF for antill6. The level of IL6 in the serum of JIA patients with treatment ineffectiveness was higher than at the beginning of disease (sJIA $52.40 \pm 73.84 \mathrm{vs} 24.4 \pm 4.7$ ( $<<0.05$ ); pJIA 36.19 $\pm 58.6 v s 2.3 \pm 0.25$ (p <0.05); OJIA $8.69 \pm 5.27 v s 1 \pm 0.1$; enthJIA $90.55 \pm 63.33 \mathrm{vs} .4 \pm 0.9 \mathrm{pg} / \mathrm{ml})$. There was no increase in IL6-level in patients with an unfavorable course of the RFpos-pJIA $(8.4 \pm 7.35 \mathrm{pg} / \mathrm{ml})$ and with uveitis $(5.59 \pm 4.94 \mathrm{pg} / \mathrm{ml})$ (norm.1.5-7pg/ml). In $57.1 \%$ of cases of RFneg-pJIA IL6 was elevated, in 3 children it was the highest (79.77$218.7 \mathrm{pg} / \mathrm{ml})$, they had anemia and osteoporosis. A high $(51-484.6 \mathrm{pg} / \mathrm{ml})$ level was observed in 4 patients with enthJIA, in 1-inadequate therapy was performed, in 3 patients there was a fever, a lag in physical development, the highest CRP (up to $300 \mathrm{mg} / \mathrm{ml}$ ). The correlation between the level of IL6 in the blood of children with JIA and the presence of lymphadenopathy in the debut of the disease $(r=0.53)$, the child's age at the debut of the disease $(r=0.63)$, examination $(r=0.74)$, and the patient's weight $(r=0.87)$, the duration from the onset of the disease to the initiation of biological therapy $(r=0.44)$, the number of exacerbations in the first years of the disease $(r=0.66-0.69)$, the formation of contractures and the limitation of movement in the joints $(r=0.75)$, radiological progression in the 1st year from the debut $(r=0.54)$, ESR $(r=0.48)$, CRP $(r=0.40)$, doctor's estimate of disease activity $(r=0.79)$, ALT $(r=0.69)$, AST $(r=0.99)$, $\mathrm{LDH}(r=0.73)$ was found. There was no correlation with the number of affected joints $(r=0.28)$, heart rate $(r=0.48)$, metabolic abnormalities on the ECG ( $r=0.39)$. In children with an adverse course of JIA, IL6 correlated backward with TNF $(r=-1)$, which was not observed in children at the onset of the disease $(r=0.19)$.

Analysis of the results of genetic examination showed that among children with treatment failure $50 \%$ had GC-allele of the IL-6-174GC gene, $27,3 \%$ had GG allele, and $23,7 \%$ had CC allele.

Conclusion: Treatment of JIA leads to changes in cytokine profile. The IL6-174CG gene is associated with a high level of IL6 in the blood. The longer the duration of the disease and the time before the start of treatment with biological DMARDs, the greater the likelihood of an increase of IL-6-level in serum. In cases of even short-term enzymemia in JIA subclinical activity with IL-6 can be suggested. In case of presence of some signs (hyperthermia, osteoporosis, anemia) in patients with antiTNF, it is advisable to determine the level of IL-6 in blood serum.

Disclosure of Interests: None declared

DOI: 10.1136/annrheumdis-2019-eular.2414

\section{AB0935 ANTI-TIF-1 G-ANTIBODIES IN JUVENILE DERMATOMYOSITIS ARE ASSOCIATED WITH VARIOUS CLINICAL PHENOTYPES}

Brigitte Bader-Meunier ${ }^{1}$, Cyril Gitiaux ${ }^{2}$, Jean-Luc Charuel ${ }^{3}$, Nicole Fabien ${ }^{4}$, Isabelle Melki ${ }^{5}$, Pierre Quartier ${ }^{6}$, Alexandre Belot ${ }^{7}$, Nathalie Streichenberger ${ }^{4}$ Christine Bodemer 6 , Audrey Aussy ${ }^{8}$, Olivier Boyer ${ }^{8} .{ }^{1}$ Hôpital Necker, Pediatrics, Paris, France; ${ }^{2}$ Hopital Necker, Pediatrics, Paris, France; ${ }^{3}$ Hôpital La Pitié, Immunology, Paris, France; ${ }^{4} \mathrm{HCL}$, Lyon, France; ${ }^{5}$ Hôpital R.Debré, Paris, France; ${ }^{6}$ Hopital Necker, Paris, France; ${ }^{7}$ HFME, Lyon, France; ${ }^{8}$ Rouen University Hospital, rouen, France

Background: Juvenile dermatomyositis (JDM) is a rare heterogeneous autoimmune disease. The identification of myositis specific antibodies (MSAs) has allowed the characterization of subgroups of JDM patients who each have specific phenotypes. Antibody $(\mathrm{Ab})$ against transcriptional intermediary factor-1- $\gamma$ (TIF-1- $\gamma$ or $\mathrm{p} 155 / 140)$ is the most common MSA in JDM ${ }^{1}$. In the American and English JDM cohorts, anti-TIF-1- $\gamma$ associated JDM is classically associated with a larger proportion of caucasians, mild or moderate severity with typical cutaneous manifestations (Gottron's papules, and/or heliotrope rash, photosensitivity), and a chronic disease course with a low mortality. The frequency of skin ulcerations and lipodystrophy differs between the two cohorts

Objectives: To report the clinical and muscle histology associations of anti-TIF-1- $\gamma \mathrm{Ab}$ in a series of patients with JDM followed in the French referral center for rare pediatric systemic autoimmune diseases.

Methods: Retrospective study of patients with JDM (according to the EULAR/ACR criteria) associated with anti-TIF-1- $\gamma$ autoantibodies and included in our CEMARA database approved by the French National Committee on Informatics and Liberty.

Results: Thirteen patients were included (males: 5, females: 8; Caucasians: 6, Black Africans: 2, North Africans: 5). Age at diagnosis ranged from 1.5 to 11 years. Serum creatine kinase was elevated in 12 patients (range: 180-43 $000 \mathrm{IU} / \mathrm{L}$ ). Three different phenotypes were identified according to the severity and course. In group $1 \quad(n=4), 3$ patients had a moderate JDM: classical cutaneous manifestations and moderate muscle involvement; relapsing course, which remitted under methotrexate/corticosteroids; an additional 2-year-old girl had an amyopathic JDM. In group 2 $(n=7)$, patients had a severe JDM : ulcerating skin lesions, severe muscle involvement, dysphagia, gastrointestinal vasculitis and/or requirement to an intensive care unit, and required more than two lines of treatment. In this group, 2/7 patients died from refractory JDM, comparing to a mortality rate of $2 \%$ in the remaining JDM patients tested for MSA and negative for anti-TIF-1- $\gamma$. Only $2 / 5$ patients achieved a complete remission under treatment. Group 3 comprised two patients with severe muscle atrophy, calcinosis and lipodystrophy and a chronic course; one of them had a very-early-onset JDM at 1.5 year-old and the other an inherited neurological involvement, potentially suggestive of a genetic predisposing condition to JDM. None of the patients developed lung involvement or malignancy. Six patients underwent a muscle biopsy which was 